Methods This cross-sectional study was carried out in the areas of two Rural Health Training Centres of Department of Community Medicine, Mahatma Gandhi Institute of Medical Sciences, Sevagram; through house-to-house visits. Two stage sampling method (30-cluster followed by systematic random) was used to reach the respondents' households. Partial correlation coefficients were used for continuous variables. Linear regression analysis was used to assess the influence of different anthropometric indicators on the systolic and diastolic blood pressure.

Results The mean systolic blood pressure was 120.2 and $118.4 \mathrm{~mm}$ $\mathrm{Hg}$ in men and women respectively while the mean diastolic blood pressure was 77.7 and $76.3 \mathrm{~mm} \mathrm{Hg}$ in men and women respectively. Mean values of body mass index (BMI), waist-hip ratio, waist circumference and waist-height ratio was significantly higher among hypertensive than normotensive men and women. There was a significant positive correlation of obesity indicators with both systolic and diastolic blood pressure; except for waist-hip ratio and diastolic blood pressure. BMI was a better predictor of both systolic $(\beta 0.59$, SE 0.11, $p<0.001)$ and diastolic blood pressure $(\beta 0.49$, SE $0.10, \mathrm{p}<0.001)$ than waist circumference.

Conclusion BMI and waist circumference had strong correlation with systolic and diastolic blood pressure.

\section{SP1-15 KNOWLEDGE, ATTITUDES AND FUNCTION OF WOMEN REFERRED TO HEALTH CENTRES IN FASA: A SURVEY OF BREAST CANCER AND SELF-EXAMINATION IN 1388 [2009/ 2010]}

doi:10.1136/jech.2011.142976m.92

S L Dehghani, ${ }^{*}$ F Rostami. Kerman University of Medical Sciences, Kerman, Iran

Introduction According to studies in Iran the incidence of breast cancer in women is increasing and it is the most prevalent cancer in women. We considered the role of self-examination and clinical inspection in order to facilitate the early detection of breast cancer and any associated reduction in mortality.

Methods This research was carried out in women referred to health centres in Fasa town. Clustered sapling was used and 300 of women were surveyed. Data collection included: age, level of education, marital status, job, family history of breast cancer, sources of information about signs of breast cancer, and information about the correct way of self-examination of breast and its importance.

Results 300 women were examined. The average age was 30 years (mode 25-30). The majority were married housewives and their education was below diploma. $40 \%$ of them knew about breast cancer and self-examination. $10 \%$ had a neutral attitude towards breast self-examination. $50 \%$ had poor function, however, as knowledge increased function also increased. Degree of knowledge was not associated with attitude.

Conclusion Most women had poor knowledge about breast cancer and poor function in self-examination. Our findings suggest it is important to focus on increasing women's knowledge levels and their ability to undertake breast self-examination.

\section{SP1-16 ASSOCIATION BETWEEN PSYCHOLOGICAL AND NUTRITIONAL PARAMETERS IN PATIENTS UNDERGOING PERITONEAL DIALYSIS}

doi:10.1136/jech.2011.142976m.93

J C N Marchette, , C R de Goés, V C Paduan, C L Mendes-Chillof, M R P Pinto, A T A Ramos-Cerqueira, J C T Caramori. Botucatu Medical School (UNESP), Botucatul Sao Paulo, Brazil

Introduction The incidence and prevalence of end-stage kidney disease has reached epidemic proportions in Brazil and worldwide, leading to high mortality. In these patients, nutritional status is associated with poorer quality of life (QoL) and health and few studies have been conducted on the subject in Brazil.

Objective Investigate the association between psychological and nutritional parameters in patients undergoing peritoneal dialysis. This is a retrospective study of 45 patients attended at the Dialysis Unit of the Clinical Hospital of Botucatu Medical School (UNESP): $58 \%$ women, mean age of 53.5 years-old, on dialysis for at least 6 months, 69\% using Automatic Peritoneal Dialysis and 63\% nondiabetic. For cognitive evaluation, the Mini-Mental State Examination (MMSE) was used and for QoL evaluation, the SF-36; for nutritional assessment, anthropometric and biochemical measurements, dietary recall and bioelectrical impedance analysis were performed.

Results Using Pearson's correlation, significant positive correlations were verified between the MMSE score and caloric and protein intake and percentage of intracellular water, and negative correlations for creatinine clearance and age; serum albumin correlated positively with the following QoL domains: physical functioning, general health, vitality, mental health and physical, social and emotional aspects; inflammatory status, measured by PCR, was negatively correlated with physical aspects and general health. In multivariate analysis, the phase angle was positively correlated with functional capacity, pain, emotional aspects and mental health.

Conclusion The association between nutritional and psychological aspects reaffirms the importance of nutritional and psychological care and the need for multidisciplinary care in this population.

\section{SP1-17 INFLUENCE OF PHYSICAL ACTIVITY INTENSITY ON ANTHROPOMETRIC INDEX AND SERUM URIC ACID CONCENTRATION IN PEOPLE WITH OBESITY}

doi:10.1136/jech.2011.142976m.94

${ }^{1} \mathrm{Y}$ Nishida, ${ }^{*} \mathrm{M}$ lyadomi, ${ }^{3} \mathrm{Y}$ Higaki, ${ }^{3} \mathrm{H}$ Tanaka, ${ }^{1} \mathrm{M}$ Hara, ${ }^{1} \mathrm{~K}$ Tanaka. ${ }^{1}$ Department of Preventive Medicine, Faculty of Medicine, Saga University, Saga, Japan; ${ }^{2}$ SUMCO Corporation, Saga, Japan; ${ }^{3}$ Laboratory of Exercise Physiology, Faculty of Health and Sports Science, Fukuoka University, Fukuoka, Japan

Introduction Physical activity (PA) is considered important in the prevention and treatment of obesity and hyperuricemia. However, scarce evidence exists regarding the influence of PA intensity on anthropometric indices and serum uric acid in people with obesity.

Methods We sampled middle-aged men with obesity and/or abdominal obesity who were employees in silicon wafer manufacture and participated in a health check-up. We examined PA using an uniaxial accelerometer, as well as measuring aerobic fitness using an electric bicycle ergometer. PA was defined as light- $[(<3$ metabolic equivalents (METs)), moderate ( $3-6 \mathrm{METs}$ ), and vigorous ( $>6$ METs). Overall METs calculated by adding the time spent at each intensity levels.

Results 71 mean took part, mean age $47.2 \pm 4.4$ years. Aerobic fitness index of lactate threshold was inversely correlated with uric acid $(\mathrm{r}=-0.26, \mathrm{p}=0.033)$, but this became non-significant after adjustment for potential confounding factors (age, BMI drinking). Light-intensity PA was inversely associated with BMI and waist circumference, even after adjustment for age and drinking (BMI $\beta=-0.543, p=0.023$; waist circumference $\beta=-1.333$, $p=0.016$ ). Both light and vigorous intensity PAs were not related to uric acid level, whereas moderate intensity PA was inversely correlated with the circulating uric acid and this remained significant, even after adjustment for age, BMI, and drinking $(\beta=-0.222$, $\mathrm{p}=0.036$ ). 
Conclusion The present results suggest that light intensity PA may have an important role in weight control while greater moderateintensity PA may be associated with lower uric acid concentration in individuals with obesity.

\section{SP1-18 ADOLESCENTS' CHRONIC DISEASES AND HEALTH COMPLAINTS: A POPULATION BASED SURVEY IN CAMPINAS, SÃO PAULO, BRAZIL}

doi:10.1136/jech.2011.142976m.95

M Braz, A de Azevedo Barros, ${ }^{*}$ M B de Azevedo Barros. State University of Campinas Medical School, Campinas, São Paulo, Brazil

Introduction The prevalence of diseases has been changing in Brazil in the last decades. The objective of this research was to estimate the prevalence of chronic diseases and health complaints among adolescents according to socioeconomic and demographic variables.

Method A cross-sectional population based survey was carried out in Campinas in the years 2008/09 (ISACamp 2008/09). Data of 929 adolescents from 10 to 19 years were obtained through household interviews. The analysis included prevalence ratio (PR) and 95\% CI. Poisson regression was used for adjusted analysis.

Results $17.54 \%$ (CI 14.34 to 21.28 ) informed having one chronic disease and $1.62 \%$ (0.92 to 2.83) having more than one. Regarding health complaints $38.47 \%$ (31.98 to 45.39 ) referred not having any problem, $33.75 \%$ (29.55 to 38.21 ) informed one problem, $17.31 \%$ (15.46 to 20.58 ) two, $7.23 \%$ (5.27 to 9.83 ) three and $3.23 \%$ (1.88 to 5.56$)$ four or more. Among the chronic diseases, asthma showed the higher prevalence: $7.59 \%$ (6.01 to 9.54). The most prevalent health complaints were: allergy $40.39 \%$ (34.90 to 46.13), frequent headache/migraine $24.83 \%$ (20.35 to 29.92), backache/ column problems $11.84 \%$ (8.97 to 15.48 ) and emotional problems (anxiety and sadness) 10.65\% (7.69 to 14.56). After adjusting for confounding the PR for chronic diseases were: 1.4 (1.06 to 1.82) for age over $15,0.68$ (0.46 to 0.99 ) for those still attending school, and 1.8 (1.06 to 3.18) for having children. The PR for health complaints were: 1.12 (1.01 to 1.24) for girls and 1.34 (1.11 to 1.62) for higher family income.

Conclusion Age from 15 to 19 years, not attending school and having children are associated with chronic diseases, and female adolescent and having a family income of over 4 minimum wages are associated with health complaints.

\section{SP1-19 DIFFUSE REFLECTANCE SPECTRAL IMAGING: A NON- INVASIVE PROMISING TOOL FOR EARLY DIAGNOSIS AND SCREENING OF MALIGNANT CHANGES IN THE ORAL CAVITY}

\section{doi:10.1136/jech.2011.142976m.96}

${ }^{1} \mathrm{M}$ M S Mathunny, ${ }^{* 2} \mathrm{~J}$ L Jayanthi, ${ }^{1} \mathrm{~V} T$ Beena, ${ }^{2} \mathrm{~N}$ Subhash. ${ }^{1}$ Department of Oral and Maxillofacial Pathology, Government Dental College, Trivandrum, Kerala, India; ${ }^{2}$ Biophotonics Laboratory, Centre for Earth Science Studies, Trivandrum, Kerala, India

Background Early detection is vital for improving the survival rate of oral cancer patients.

Materials and Methods Spectral data at 545 and $575 \mathrm{~nm}$ in diffuse reflectance (DR) spectra were imaged from oral lesions of 55 patients and 103 sites of 23 healthy volunteers with an electron multiplying charge coupled device (EMCCD) camera. A computer-generated algorithm based on pixel intensity of DR image ratio (R545/R575) was used for pseudo-colour mapping the entire oral lesion. Tissue biopsies of all patients were taken from selected sites with maximum malignant potential. Spectral data of ratio image were then compared against the histopathology results.

Results The mean age of patients and healthy volunteers were $55.7 \pm 12.0 \mathrm{yrs}$ and $28 \pm 7.0 \mathrm{yrs}$, respectively. Majority of patients were tobacco users (43/55), while 23/55 were users of alcohol. The median pixel intensity value of the ratio-image was significantly lower $(p<0.001)$ in the healthy group $(0.87$, IOR: $0.82-0.94)$ in comparison to pre-malignant (1.35, IOR: 1.13-1.67) and malignant group (2.44, IOR: 1.78-3.80). The area under the ROC curve (AUC) of healthy to pre-malignant, healthy to malignant, pre-malignant to malignant and healthy to pre-malignant/malignant comparisons were 0.94 (95\% CI 0.86 to 1.00), 0.99 (95\% CI 0.99 to 1.00 ), 0.84 (95\% CI 0.73 to 0.95 ) and 0.97 (95\% CI 0.94 to 1.00), respectively. The discriminant function analyses with leave-one-out classification differentiate healthy from pre-malignant/malignant lesions correctly in $84.2 \%$ occasions after cross-validation (Wilks' $\lambda=0.744, \chi^{2}=45.97$ with degree of freedom $=1, \mathrm{p}<0.001$ ).

Conclusion DR spectral imaging efficiently discriminates healthy tissues from oral malignant and pre-malignant lesions.

\section{SP1-20 PREVALENCE OF COGNITIVE IMPAIRMENT AND DEPRESSION AND ASSOCIATED FACTORS IN ELDERS LIVING IN LONG-STAY INSTITUTIONS IN BRAZIL}

doi:10.1136/jech.2011.142976m.97

${ }^{1} \mathrm{~L}$ M Santiago, ${ }^{*}{ }^{1} \mathrm{~L}$ L Luz, ${ }^{1,2} \mathrm{~J}$ F Santos-Silva, ${ }^{1,3} \mathrm{P}$ H Oliveira, ${ }^{1} \mathrm{~L}$ C Alves, ${ }^{1} \mathrm{E}$ E Mattos. ${ }^{1}$ Oswaldo Cruz Foundation, National School of Public Health, Rio de Janeiro, Brazil; ${ }^{2}$ Health Secretariat of Mato Grosso do Sul State, Mato Grosso do Sul/MS, Brazil; ${ }^{3}$ Health Secretariat of Mato Grosso State, Mato Grosso/MT, Brazil

Introduction Cognitive impairment and depression are among the health conditions that affect the ability of the elders to live independently, leading to their placement at long-stay institutions. This phenomenon has been increasing in Brazil. This study analyzes the prevalence of these health conditions and its associated factors in elders who live at these institutions.

Methods This is a cross-sectional study with elderly residents of long-stay institutions in four Brazilian cities. The study's questionnaire included socio-demographic and health-related variables and the assessment of functioning, cognition and mood with scales widely used in elderly populations. The population profile was characterised and the prevalence of cognitive impairment and depression was estimated. Bivariate and multivariate analyses were performed with Poisson regression.

Results These are preliminary results for 340 elders. The mean age was 75.5 years, most were men, with $0-4$ years of schooling and $<5$ years of institutionalisation. The prevalence of cognitive impairment was $65.8 \%$ and of depression $49.1 \%$. Variables associated to cognitive impairment were depression (RP 4.39; 95\% CI 1.11 to 7.27 ) and female gender (RP 2.96; 95\% CI 1.28 to 6.84). The referral of very poor/poor health (RP 2.69; 95\% CI 1.37 to 5.27), hospitalisation in the last year (RP 1.55; 95\% CI 1.04 to 2.33) and presence of cognitive impairment (RP1.42; 95\% CI 1.11 to 1.83 ) were associated to a high probability of depression.

Conclusions The high prevalence of these two conditions in elders living at long-stay institutions in Brazil denotes the importance of health policies aiming to improve the quality of care received by this population group. 